\title{
Las redes sociales en el sector artesanal de Oaxaca-México y Plaza Matilde en Loja-Ecuador, como herramienta de comunicación para publicitar emprendimientos
}

\section{Social networks in the artisanal sector of Oaxaca-Mexico and Plaza Matilde in Loja-Ecuador, as a communication tool for public enterprises}

Irma Abrigo-Córdova

Yolanda Morales Espinoza

Universidad Internacional del Ecuador, Ecuador

Cristina Romero Rojas

Universidad de la Sierra Sur, México

Autor para correspondencia: irmabrigocordova2016@gmail.com, cristinaromerorojas@gmail.com, dimoraleses@internacional.edu.ec

Fecha de recepción: 11 de Octubre 2017 - Fecha de aceptación: 15 de Febrero de 2018

Resumen: La presente investigación es un trabajo interdisciplinario desarrollado en el sector artesanal de Oaxaca-México y Plaza Matilde en Loja-Ecuador, tiene por objeto determinar la importancia de las redes sociales como herramienta de comunicación para publicitar emprendimientos artesanales. A través de la técnica grupos focales y entrevista, los artesanos de Loja en Plaza Matilde expresan que las redes sociales están siendo subutilizadas, pues, no detallan las características y presentaciones de los productos ofertados. En Artesanías oaxaqueñas de Oaxaca- México, la página de Facebook: Artesanías Oaxaqueñas se observa que fue creada en noviembre de 2016 y que desde enero de 2017 no ha tenido actividad alguna, además, no cuenta con imágenes de las artesanías que venden. En esta página se exportan productos artesanales a cualquier parte del mundo, con una garantía excepcional y cuentan con productos de calidad con precios accesibles. Sin embargo, solo cuenta con 381 personas que les gusta la página y 385 personas lo siguen. En comparación a las personas que siguen los comentarios, las veces compartidos, los "me gusta" son pocos (menores a 20) y no se evidencia publicaciones de los productos que producen. Se plantean redes sociales interactivas con una metodología que permitirá posesionar los emprendimientos artesanales de las localidades citadas, logrando la vinculación internacional entre cada una de ellas.

Palabras Clave: colaboración; emprendimiento; interdisciplinariedad; medios sociales; publicitario

Abstract: This research is an interdisciplinary work developed in the artisanal sector of OaxacaMexico and Plaza Matilde in Loja-Ecuador, aims to determine the importance of social networks as a communication tool to advertise craft enterprises. Through the technique focus groups and interview, the artisans of Loja in Plaza Matilde express that the social networks are being underutilized, therefore, they do not detail the characteristics and presentations of the products offered. In Artesanías oaxaqueñas of Oaxaca - Mexico, the page of Facebook: Artesanías Oaxaqueñas is observed that was created in November of 2016 and that from January of 2017 has 
had no activity some, in addition, does not count with images of the crafts that they sell. On this page, handcrafted products are exported to any part of the world, with an exceptional guarantee and quality products with affordable prices. However, it only has 381 people who like the page and 385 people follow it. Compared to the people who follow the comments, the times shared, the "like" are few (less than 20) and there is evidence of publications of the products they produce. Interactive social networks are proposed with a methodology that will allow the artisanal enterprises of the towns mentioned to be possessed, achieving the international link between each one of them.

Key Words: social media; interdisciplinarity; collaboration; advertising; entrepreneurship

\section{Problema o argumento}

\section{Introducción}

El proyecto se realizó en los sectores artesanales de Oaxaca-México y Plaza Matilde en Loja-Ecuador, se originó como una iniciativa de la red de contactos orientada a trabajar con herramientas de comunicación, siendo la finalidad, publicitar emprendimientos artesanales para incrementar las ventas.

\section{Revisión de literatura}

La investigación presentada se constituyó en un trabajo académico interdisciplinario entre docentes de la Facultad de Ciencias Administrativas de la Universidad Internacional del Ecuador extensión Loja y Docente de la Carrera de Informática de la Universidad Sierra Sur de OaxacaMéxico, se trata del primer avance del proyecto, presentado con la finalidad de realizar acercamientos en los sectores artesanales de Oaxaca-México y Plaza Matilde en Loja-Ecuador, para determinar la importancia de las redes sociales como herramienta de comunicación en la publicidad de emprendimientos e incrementar las ventas, para el efecto se realizó la visita a los sectores investigados logrando identificar algunas necesidades entre ellas, la subutilización de las redes sociales.

El presente trabajo se realizó el estado del arte con el objetivo de enfocar la investigación, considerando entre los aspectos principales, las redes sociales, siendo "un lugar de interacción virtual que sirve como punto de encuentro para varios miles o incluso millones de personas de todo el mundo, que comparten unos intereses y/o características sociodemográficas, y que participan en un proceso de comunicación...". (Gómez \& Otero, 2013). En este contexto se evidencia que las redes sociales disponen de millones de usuarios activos, por lo cual, las empresas han tomado ventaja para posicionarse en el mercado.

Las empresas a través de las redes sociales se convierten en fuente de información para los residentes, anunciando las actividades que realiza, dándose a conocer a compradores y posibles clientes y logrando acercarse a ellos a través de la comunicación. Constituye de gran importancia que los artesanos estén presentes en las redes, de forma profesional, lo cual les permite promocionar sus productos, su capacidad artística o académica, el territorio donde ejerce su oficio, etcétera (Suárez, 2016). Según (Gómez \& Otero, 2013) plantea las siguientes redes sociales más utilizadas. 
Facebook es una de las redes sociales más utilizadas, considerada como "herramienta que acerca considerablemente clientes y empresas, facilitando su interacción, se logra el objetivo de la publicidad, se expone de manera rápida la opinión de sus clientes, información de ellos e interactúan de una manera rápida y directa” (Gálvez, 2015)

Utilizar las redes sociales es estratégico para publicitar los emprendimientos artesanales, "en este sentido la publicidad en internet ha incorporado nuevas plataformas y oportunidades de negocio para las empresas y marcas..." (Gañán, 2015). Al respecto, también se debe considerar que todo emprendimiento nace de una idea, con el propósito de conseguir independencia y estabilidad económica; y como respuesta a "los altos niveles de desempleo y la baja calidad de los empleos existentes, han creado en las personas, la necesidad de generar sus propios recursos de iniciar sus propios negocios, y pasar de ser empleados a ser empleadores" (Jiménez, 2013)

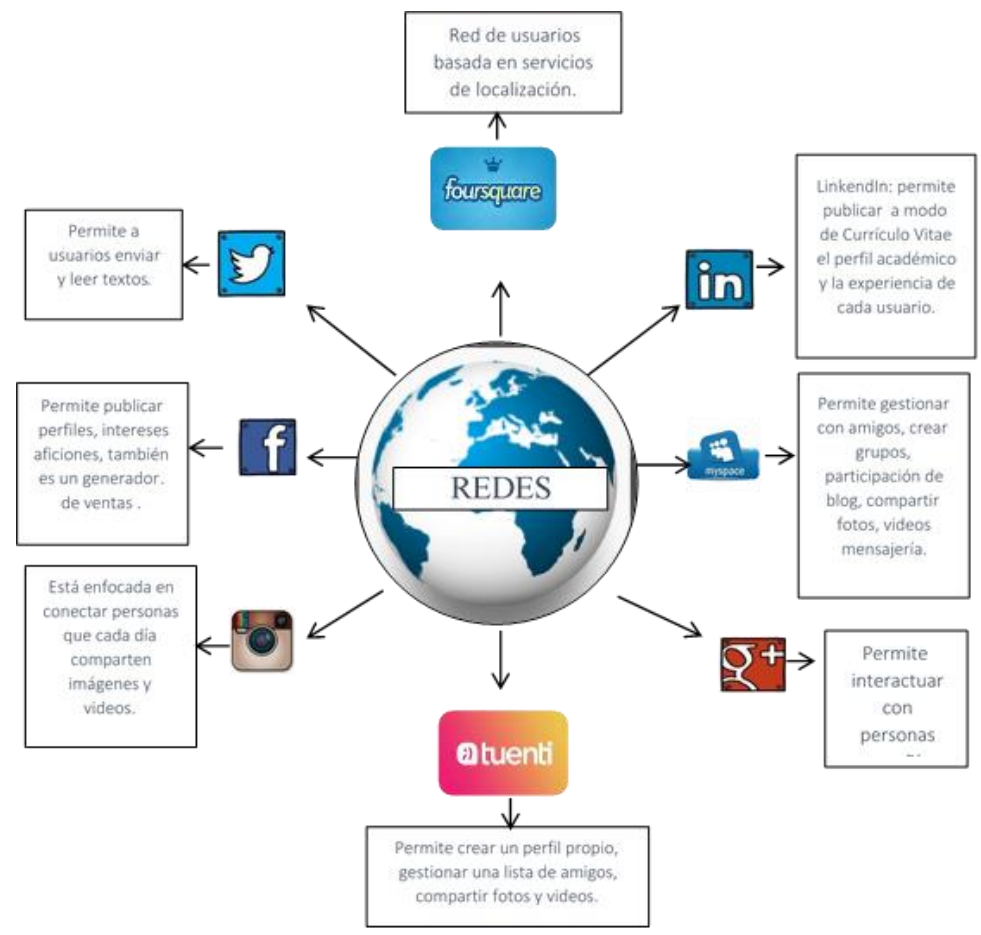

Cuadro $N^{\circ} 01$ : Redes sociales más utilizadas.

Fuente: Datos tomados de Redes sociales en la empresa (2013).

\section{Finalidad}

En la investigación planteada se propuso los siguientes objetivos:

\section{Objetivo general:}

Determinar la importancia de las redes sociales en el sector artesanal de Oaxaca-México y Plaza Matilde en Loja-Ecuador, como herramienta de comunicación para publicitar emprendimientos.

\section{Hipótesis:}

Las redes sociales en el sector artesanal de Oaxaca-México y Plaza Matilde en LojaEcuador se constituyen en importantes herramientas de comunicación para publicitar 
emprendimientos.

\section{Variables}

Variable dependiente: Redes sociales. Variable independiente: Emprendimientos.

\section{Metodología}

La metodología de ésta investigación, se fundamenta principalmente en la epistemología cualitativa, descrita a continuación:

1. Investigación del fundamento teórico de redes sociales, relación de estas con las empresas y el por qué deben estar los artesanos en las redes sociales; la importancia de la publicidad en las redes sociales; el emprendimiento, su importancia y características básicas de los emprendedores; las artesanías, ventajas de las actividades artesanales y características de la producción artesanal; ventaja de la competitividad y elementos clave de esta.

2. Aplicación de las técnicas cualitativas: grupos focales y observación directa en tiendas o plazas artesanales de Ecuador y México.

3. Creación y/o rediseño de una página de Facebook en una de las redes sociales más populares.

4. Seguimiento, análisis y documentación de los resultados.

5. Discusión de los resultados, conclusiones y líneas abiertas de investigación.

\section{Resultados}

Los resultados obtenidos hasta el momento, siguiendo la metodología establecida son los siguientes:

- Investigación del fundamento teórico de los términos siguientes: redes sociales, publicidad, emprendimiento, artesanías, competitividad. Considerando características y puntos clave de cada uno de ellos.

- Aplicación de las técnicas cualitativas: grupos focales en plaza Matilde de Loja, Ecuador y observación directa en artesanías oaxaqueñas de Oaxaca, venta de sus productos.

- Cabe mencionar que la investigación se desarrolló en el Centro artesanal Plaza Matilde de la ciudad de Loja-Ecuador, en la cual se aplicó la técnica de grupos focales a líderes de diferentes emprendimientos, quienes ofertan los siguientes productos y servicios: elaborados en chaquiras, limpieza, chocolatería en general, manualidades y bordados, confección textil y tejidos, artesanías pintadas y armadas en cuero, productos orgánicos. bisutería.

- Un primer análisis para el rediseño de la página de Facebook de plaza Matilde (recomendaciones para la publicidad de sus artesanías e incremento en sus ventas).

- Artesanía oaxaqueña cuenta con una página de Facebook, donde se observa inactividad desde noviembre de 2016, dejando en espera de una respuesta a los interesados que comentan sus dudas para la compra de las artesanías ofertadas. Por lo que se ha llevado acabo un primer análisis para iniciar la publicidad profesional en la página de Facebook.

- En el caso de la tienda de artesanías Tlalchinoltepetl de México, se creó la página de Facebook para la publicidad de sus productos, para ello se aplicó la técnica de grupos 
focales. Además, de contar con el apoyo del equipo profesional de fotografía del joven originario de Chocamán, Dual Ramírez Vega. Las primeras pruebas han tenido resultados positivos. Estamos en proceso de administrar el contenido para posteriormente analizar los resultados.

- Además de que se ha mostrado interés por parte de otros artesanos oaxaqueños y veracruzanos para utilizar las redes sociales, en específico Facebook, para la publicidad.

- En artesanías oaxaqueñas de Oaxaca, se utilizó la técnica de observación directa de los diferentes elementos de la red social, las artesanías que destacan principalmente son: vestimentas típicas con diferentes técnicas de bordado y tapetes elaborados de lana, sin embargo la página con la que cuentan no tiene contenido de publicidad.

- Cabe mencionar que el grupo de artesanos Tlalchinolpetl de Chocamán, Veracruz, México; está interesado en sumarse a nuestra investigación como estudio de caso, ellos no contaban con ningún tipo de publicidad inclusive en las redes sociales.

La técnica de grupos focales es un espacio de opinión para captar el sentir, pensar y vivir de los individuos, provocando auto explicaciones para obtener datos cualitativos. Kitzinger lo define como una forma de entrevista grupal que utiliza la comunicación entre investigador y participantes, con el propósito de obtener información. Para Martínez-Miguelez, el grupo focal "es un método de investigación colectivista, más que individualista, y se centra en la pluralidad y variedad de las actitudes, experiencias y creencias de los participantes, y lo hace en un espacio de tiempo relativamente corto". La técnica es particularmente útil para explorar los conocimientos y experiencias de las personas en un ambiente de interacción, que permite examinar lo que la persona piensa, cómo piensa y por qué piensa de esa manera. El trabajar en grupo facilita la discusión y activa a los participantes a comentar y opinar aún en aquellos temas que se consideran como tabú, lo que permite generar una gran riqueza de testimonios. (Hamui Sutton \& Varela Ruiz, 2013)

Se encuentran otras técnicas similares al grupo focal, que se pueden diferenciar por sus particularidades para responder a las preguntas de investigación. Así, la observación directa es más apropiada para estudios de roles sociales en una organización, en tanto, que el grupo focal es particularmente sensible para el estudio de actitudes y experiencias. (Hamui Sutton \& Varela Ruiz, 2013)

\section{Discusión}

Se empleó la metodología cualitativa, aplicando técnicas de recolección de la información: observación, entrevista y grupos focales. La presente investigación permitió conocer que las redes sociales en los centros artesanales están siendo subutilizadas y determinó la importancia de las mismas como herramienta de comunicación para publicitar emprendimientos.

Se rediseñó una nueva página de Facebook para plaza Matilde con el objetivo de promover sus productos para el incremento de sus ventas. Para el efecto se contaron con las fotografías de las artesanas y sus productos. 
En lo referente al centro artesanal de Oaxaca, a recaudación de fotografías y descripción para el contenido de la página de Facebook Artesanías Oaxaqueñas está en proceso. En el contenido de la página de Facebook se ubicará la historia o parte de la biografía de cada uno de los artesanos, para que sean reconocidos a nivel internacional. Es necesario incluir en la investigación y apoyar a los artesanos interesados de Oaxaca, Oaxaca y Chocamán, Veracruz ambos de México. Ya se tuvo acercamiento con ellos, aplicando ambas técnicas: grupos focales, observación directa y entrevista.

Se espera alcanzar los objetivos principales de esta investigación: publicidad de las artesanías en la red social Facebook, incremento en ventas directas artesano-comprador, alcanzar la competitividad de los grupos de artesanos y generar un intercambio de artesanías de México y Ecuador, como continuación de la propuesta. En este primer avance, se ha identificado la subutilización de las redes sociales en el sector artesanal de Oaxaca-México y Plaza Matilde en Loja-Ecuador; y se ha determinado la importancia de las mismas, como herramienta de comunicación para publicitar emprendimientos.

\section{Conclusiones}

Se determina la importancia de las redes sociales como herramientas de emprendimiento en los sectores artesanales, por las características que poseen, como: fácil acceso, concentración de mercado, análisis de datos estadísticos, etc. Siendo utilizadas en diferentes ámbitos y sectores de nuestra vida, por lo que deben emplearse para apoyar a los sectores mencionados, buscando el beneficio directo del mismo, considerando las ventajas de la competitividad y principios de marketing de Facebook.

La adaptación de la tecnología en beneficio al desarrollo, enriquecimiento cultural y económico e intercambio de países centroamericanos en un inicio Ecuador-México, puede lograrse mediante las redes sociales como Facebook. Aplicando técnicas de emprendimiento para los elementos clave de ventaja competitiva, se puede lograr una percepción real en el mercado artesanal para ser diferentes, únicos y tener una determinación en cuanto a ventas alcanzadas.

\section{Bibliografía}

Besares, C. M. (2002). Sitio web Madre Fértil. Recuperado el 22 de abril de 2017, de http://www.oni.escuelas.edu.ar/2002/SANTIAGO_DEL_ESTERO/madre-fertil/produca.htm

Gálvez Clavijo, I. (2015). Facebook para empresas y emprendedores. IC Editorial.

Gálvez Clavijo, I. (2015). Facebook para empresas y emprendedores. IC Editorial.

Gomez Vieites, A., \& Otero Barros, C. (2013). Redes sociales en la empresa. Bogota: Editorial Ra-ma.

Gomez Vieites, A., \& Otero Barros, C. (2013). Redes sociales en la empresa. Bogota: Ediciones de la u. 
Guzmán, U. (02 de mayo de 2007). The Cult. Recuperado el abril de 2017, de http://www.thecult.es/Tendencias/historia-de-la-publicidad/La-publicidad-a-partir-de-laEdad-Media.html

Hamui Sutton, A., \& Varela Ruiz, M. (2013). La técnica de grupos focales. Elsevier, 2, 55-60.

Jimenez Jimenez, A. (29 de JULIO de 2013). ISSUU. Recuperado el 22 de ABRIL de 2017, de https://issuu.com/alejandra.jimenez.8a/docs/emprendimiento_presentacion_corregi

Jotapgarrido. (13 de agosto de 2012). Antoempleos Sostenibles. Recuperado el 23 de abril de 2017, de http://www.autoempleosostenible.com/tienen-los-artesanos-un-tratamiento-legal-especial/

Luna Correa, J. E. (Septiembre de 2012). Eumed.net. Recuperado el 21 de abril de 2017, de Eumed.net: http://www.eumed.net/tesis-doctorales/2013/jelc/jelc.pdf

Montiel, D. (s.f.). elblogdedany. Recuperado el 21 de abril de 2017, de https://elblogdeldany.wordpress.com/

Osorio Tinoco, F., Murillo Vargas, G., \& González Ocampo, C. H. (enero 2015). Emprendimiento, Redes e Innovación. Programa Editorial Universidad del Valle.

Porter, \& Porter, M. E. (2008). Ventaja Competitiva. En Porter, \& M. E. Porter, Estrategias competitivas: Los conceptos centrales (pág. 20). México: Compañia Editorial Continental, S.A De C.V Mexico.

Porto Perez, J., \& Gardey, A. (2013). Definición.de: Defenición de emprendimiento. Recuperado el 21 de abril de 2017, de http://definicion.de/emprendimiento/

Santana, U. (16 de agosto de 2012). partesanal.blogspot.com. Recuperado el 21 de abril de 2017, de http://partesanal.blogspot.com/2012/08/artesanal.html

Suárez Martín, A. (20 de enero de 2016). artempleo. Recuperado el 23 de abril de 2017, de https://artempleo.wordpress.com/2016/01/20/por-que-deben-estar-los-artesanos-en-las-redessociales-2/

Valls Arnau, M. (enero 2016). Las redes sociales: herramienta de gestion empresarial. Urgerman Editor.

Victor, G. (Julio de 2015). Blog Servilia. Recuperado el 23 de abril de 2017, de http://blog.servilia.com/2015/07/27/la-importancia-de-la-publicidad-en-las-redes-sociales/

Vilajoana, A. S., Jimenez Morales, M., \& Gonzalez Romo, Z. (enero 2017). Como aplicar los conceptos basicos de publicidad. Editorial UOC.

Villafaña Figueroa, R. (s.f.). Sities.google.com. Recuperado el 26 de abril de 2017, de https://sites.google.com/site/competenciaestrategia/ricardo-villafana-figueroa

Villalba Quesada, C. (enero 2003). Redes sociales: un concepto con importantes implicaciones en la intervencion comunitaria. Colegio Oficial Psicologos de Madrid. 